\title{
The defect of blood coagulation in joints
}

\author{
A. J. HARROLD \\ From the Institute of Orthopaedics, Royal National Orthopaedic Hospital, \\ Brockley Hill, Stanmore, Middlesex, and St. Mary's Hospital, London
}

SYNOPSIS Clinical and experimental observations are described that indicate that intra-articular fibrinogenolysis is a cause of the defect of coagulation seen when blood is extravasated into a synovial joint.

The observation that blood shed into synovial joints apparently fails to clot has aroused interest for many years (Jaffe, 1897; Julliard, 1902; Kling, 1938; Ropes and Bauer, 1953). Studies on the behaviour of intra-articular fractures (Harrold, 1960a) have suggested that the fluid state of extravasated blood in joints may be one reason for the preservation of the joint space after injury and for the perpetuation of the fracture gap in intra-articular fractures. On this account a study of haemarthroses was undertaken, with particular reference to the factors that interfere with the clotting of blood in joints.

\section{CLINICAL OBSERVATIONS}

Although in clinical experience fluid blood may be obtained by aspiration or seen to flow out of the joint at operation, intra-articular clots are often found. Twenty-five traumatic haemarthroses were carefully inspected at operation. The findings are set out in Table I. Small soft clots were found in

\section{TABLE I}

CLINICAL HAEMARTHROSES

\begin{tabular}{|c|c|c|c|c|}
\hline \multicolumn{2}{|c|}{ Duration of haemarthrosis $<24 \mathrm{hr}$. } & \multirow{2}{*}{$\frac{1-2 \text { da.ys }}{5}$} & \multirow{2}{*}{$\frac{3-7 \text { days }}{5}$} & \multirow{2}{*}{$\frac{>7 \text { days }}{6}$} \\
\hline Number of haemarthroses & 9 & & & \\
\hline No clot found & 5 & 3 & 2 & 5 \\
\hline Small clots found & 3 & 1 & 2 & 1 \\
\hline Over a quarter volume & 1 & 1 & 1 & 0 \\
\hline Over half volume clotted & 0 & $\mathbf{0}$ & 0 & 0 \\
\hline
\end{tabular}

nearly half the cases. They were seen floating free or attached to injured surfaces and they did not adhere to healthy synovial membrane or to cartilage. Joints opened later than two weeks from injury were usually quite 'clean', with no trace of coagulated blood. The defect of blood coagulation was thus frequently incomplete.

Received for publication 30 December 1960

\section{EXAMINATION OF HAEMARTHROSIS FLUIDS}

The fluid component of seven human haemarthroses was examined. The haemoglobin concentration varied from 7.3 to $18.8 \mathrm{~g} . / 100 \mathrm{ml}$., and averaged 11.0 g. $/ 100 \mathrm{ml}$. All specimens contained mucin, none contained fibrinogen, and considerable haemolysis was seen in all. An antithrombin titration, by the method of Biggs and Macfarlane (1957), was performed on two specimens; no excess of antithrombin compared with normal human plasma was demonstrated. These specimens were also tested for free protease on a heated bovine fibrin plate (Astrup and Müllertz, 1952), with negative results.

The fluid component of a rabbit haemarthrosis, induced by injecting blood two hours before, was also examined. The haemoglobin concentration was identical with that of the heart blood injected, namely, $10 \cdot 3 \mathrm{~g} . / 100 \mathrm{ml}$. Slight haemolysis was noted. The fibrinogen level was $2.3 \mathrm{mg}$. $/ 100 \mathrm{ml}$., that of the plasma being $263 \mathrm{mg} . / 100 \mathrm{ml}$. There was no excess antithrombin and no free protease.

The fact that there was no excess antithrombin and no fibrinogen suggested that it was improbable that blood in joints failed to clot because of interference with the generation of thrombin or its action. These observations were, however, compatible with either blood coagulation followed within three or four hours by clot lysis, or with destruction of fibrinogen before clotting could occur.

\section{EVIDENCE AGAINST RAPID CLOT LYSIS}

A series of experiments in dogs tested the possibility that the fluid state of haemarthroses was because of the blood clotting followed by rapid clot lysis.

Mongrel dogs, 25-30 lb. (11-3-13.6 kg.) in weight and not more than 3 years old, were anaesthetized with Nembutal. Haemarthroses were induced by injecting 1.0 to $10.0 \mathrm{ml}$. freshly drawn venous blood 
into the knee and shoulder joints, and by passing a $3 \mathrm{~mm}$. drill across the elbow joints. Siliconed or paraffined all-glass syringes with size 12 needles were used. The joints were opened at intervals and the state of the contents determined by inspection.

In eight haemarthroses caused by drilling across the joint, and explored five, 10, 20, 40,60 minutes and two, three, and four hours later, bloody fluid with no clot was discovered. In 17 haemarthroses produced by injecting blood, and explored 10, 15, 20, 30, 40, 60 minutes, and five and six hours later, partial clotting was observed in 14 . The joints in which no clot formed were three of five that received only $1.0 \mathrm{ml}$. blood. There was no relationship between the quantity of clot found and the time since the haemarthrosis started and therefore no suggestion that clot lysis was occurring during the period of observation.

\section{FIBRINOGENOLYSIS IN JOINTS}

The evidence for fibrinogenolysis in synovial joints has already been described (Harrold, 1960b). When citrated plasma was injected into the knee of an anaesthetized rabbit, 3 to 4 months old, serial estimations showed a fall of fibrinogen level to zero within one and a quarter hours, a fall which was appreciably slower in fully grown rabbits (Fig. 1).

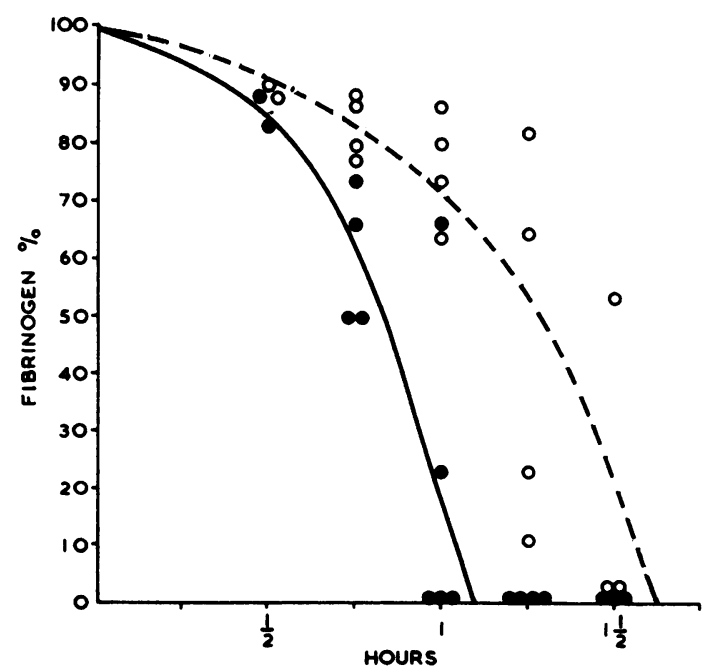

FIG. 1. Fibrinogen levels of citrated plasma in the rabbit knee. The fibrinogen concentration is expressed as a percentage of the maximum. The solid dots, averaged by a continuous line, represent the results of five experiments in rabbits less than 8 months old. The open dots, averaged by a broken line, are derived from four experiments in rabbits over 11 months old.
It was not accompanied by the formation of an. equivalent amount of fibrin clot. Fibrinogenolysi was not seen in the recently dead animal. It was inhibited by heparin, 5 units $/ \mathrm{ml}$., and by soy bears trypsin inhibitor, $1.0 \mathrm{mg} . / \mathrm{ml}$.

A quantitative study of blood clot formation ir joints was carried out to see if there were anf correlation between the features of fibrinogenolysis and the defect of blood clotting in joints.

Preliminary experiments in vitro were necessary.

FACTORS INFLUENCING BLOOD CLOT WEIGHT IN VITR冬 Rabbit blood was collected by heart puncture into $10 \mathrm{ml}$. siliconed syringe, a two-syringe technique being used. One millilitre aliquots were immediately measuredo from the syringe into a series of $1.0 \times 7.5 \mathrm{~cm}$. test-tubesw in a water-bath at $37^{\circ} \mathrm{C}$. After a set period the clot wasr gently dislodged from the sides of each tube, washed oup with $2.0 \mathrm{ml}$. normal saline and filtered through a single layer of cotton gauze in a conical funnel. Filtration was for 15 minutes under cover to limit evaporation. The clot was then gently lifted off with forceps and weighed

EFFECT OF TIME ON CLOT WEIGHT IN VITRO When rabbit: blood was allowed to clot in plain glass tubes the weigh of clot obtained did not reach a steady maximum untip about two hours had passed (Fig. 2). Furthermore, the filtrates obtained in the first three-quarters of an houp themselves clotted. Thus, although the blood had ap? parently clotted within 10 minutes, a much longeฉ period was needed for the process to go to completion $\overrightarrow{\bar{\delta}}$ Similar findings were obtained when the experiment was repeated with human blood.

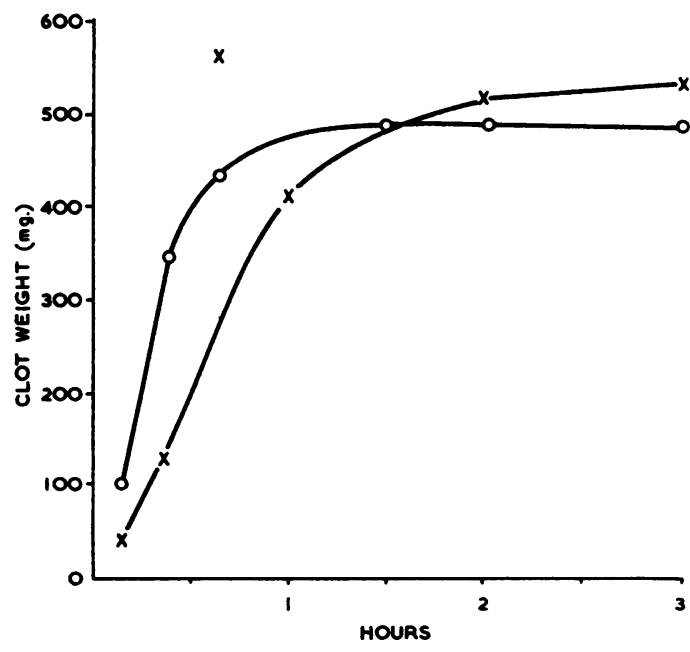

FIG. 2. Weights of clot obtained from $1.0 \mathrm{ml}$. blooळ clotting in plain glass tubes at $37^{\circ} \mathrm{C}$. Rabbit blood (o- of and human blood $(\times-\infty)$ were used. The exceptionot third clot weight in the experiment with human blood mag be attributed to contamination of the test tube. 
EFFECT OF SPEED OF COAGULATION ON CLOT WEIGHT IN VITRO Standardized human brain thromboplastin giving a one-stage prothrombin time on normal human plasma of 15 to 20 seconds was prepared, and $0.1 \mathrm{ml}$. placed in the test-tube before adding the blood. A large weight of clot rapidly formed, decreasing to a steady level over the following hour. The final clot weight was greater, in four experiments, by an average of $18 \%$ over that obtained when no thromboplastin was added. Conversely, if clotting were delayed by placing the blood in siliconed tubes, the final clot weight was less by $15 \%$.

EFFECTS OF DILUTION OF BLOOD ON CLOT WEIGHT IN VITRO When rabbit blood was diluted with saline, serum, or plasma the final clot weight obtained from $1.0 \mathrm{ml}$. diluted blood was directly proportional to the red cell content of the mixture. Diluting the blood with a suspension of red cells in saline had little effect on the clot weight up to $50 \%$ dilution but had an increasing effect after this (Fig. 3).

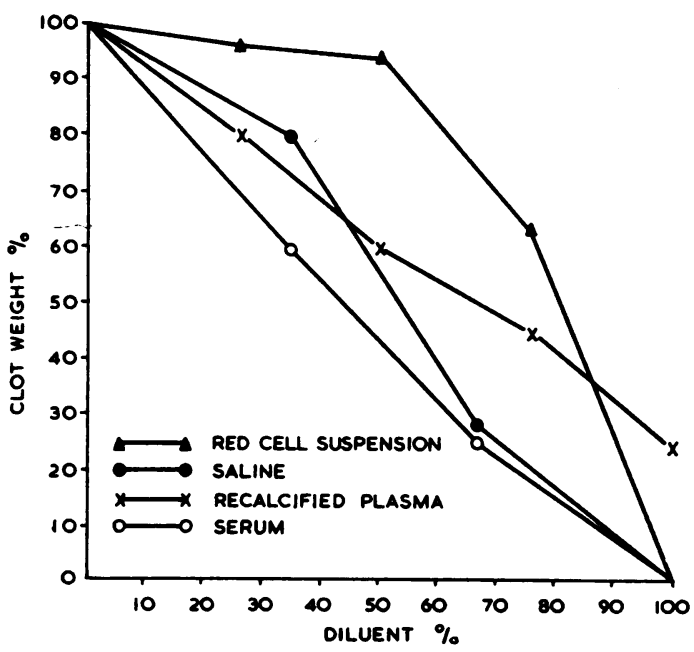

FIG. 3. Effect of dilution on weights of blood clot.

\section{CLOTTING DEFECT IN THE RABBIT KNEE JOINT}

With the rabbit anaesthetized with Nembutal, $1.0 \mathrm{ml}$. heart blood was withdrawn into a paraffined, all-glass Mantoux syringe and immediately injected through a size 20 needle already placed in the knee. A control in vitro was set up at the same time, using plain glass tubes without added thromboplastin. After two hours the animal was killed and the joint explored. Clot was gently removed with forceps and the joint washed out with $2.0 \mathrm{ml}$. saline. Filtration and clot weighing in the standard manner followed. The clot, filtrate, and filter gauze were then mechanically mixed and broken up. Distilled water was added to $10.0 \mathrm{ml}$. and the haemoglobin concentration of the supernatant estimated. From this the total haemoglobin in the joint was estimated. Knowing the amount of haemoglobin in $1.0 \mathrm{ml}$. heart blood, the clot weight obtained was corrected to the weight per millilitre of blood, thus allowing for either the effect of further bleeding into the joint, or more often, for leakage of blood out of the joint along the needle track and down the tibialis anterior tendon sheath.

The results in eight experiments in rabbits 3 to 4 months old are given in Table II. On average the corrected joint clot weights were $60 \%$ of those of the controls in vitro.

TABLE II

DEFECT OF CLOT WEIGHT IN JOINTS

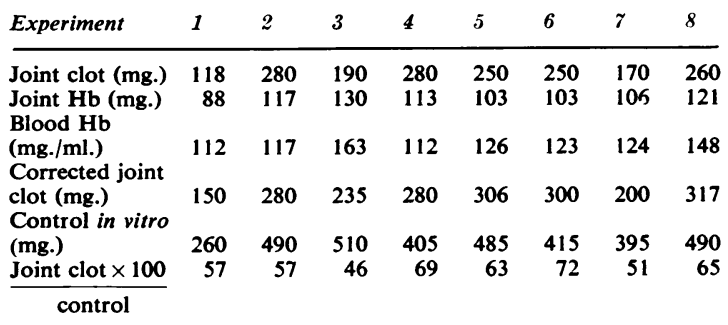

Average correction applied to joint clot weight $-16.5 \%$ increase Corrected joint clot weight averaged $60.0 \%$ control

\section{CORRELATION OF JOINT FIBRINOGENOLYSIS WITH CLOTTING DEFECT}

Fibrinogenolysis occurred more slowly in old rabbits than young. The corrected joint clot weights in three rabbits 14,7 , and 10 months old were 66,89 , and $97 \%$ of the controls respectively, averaging $84 \%$.

Fibrinogenolysis takes time, and if clotting is accelerated by adding thromboplastin, the effect of fibrinogenolysis on clot weight should be much reduced. The weights were compared of clot obtained from $1.0 \mathrm{ml}$. blood in vitro and in a joint when $0.1 \mathrm{ml}$. standardized thromboplastin had been added to the tube and to the joint immediately before the blood. In four experiments the corrected joint clot weights were $75,82,98$, and $96 \%$ of the controls, averaging $88 \%$.

Fibrinogenolysis does not occur after death. One millilitre of blood was injected into one knee of an anaesthetized rabbit. After two hours the joint was opened and the clot collected in the usual way. Just before arthrotomy $1.0 \mathrm{ml}$. blood was injected into the opposite knee and the animal immediately killed. Body temperature was maintained in the hot room at $37^{\circ} \mathrm{C}$. After a further two hours the second knee was opened and the clot extracted and weighed. The joint clot weights were compared with those of controls in vitro. In four experiments the dead joint clot weights were 81, 120,64 , and $96 \%$ of the controls, averaging $90 \%$. The living joint clot weights were $58,68,48$, and $65 \%$ respectively (average $60 \%$ ).

\section{DISCUSSION}

These experiments support the view that fibrinogenolysis is responsible for at least part of the defect of clot formation noted when blood coagulates in a 
synovial joint. Fibrinogenolysis takes at least one hour to go to completion, but the conversion of fibrinogen to fibrin in the absence of added thromboplastin has also been shown to be a relatively slow process.

It is possible that, as a result of fibrinogenolysis, secondary anticoagulant factors appear (Stormorken, 1957; Triantaphyllopoulos, 1958; Niewiarowski, Latallo, and Stachurska, 1959) which, by delaying clotting, favour more fibrinogenolysis. These factors are, however, labile and disappear with further proteolysis.

The presence of plasmin activator in joint tissues (Astrup and Sjølin, 1958; Lack, 1959), the inhibitory effect of heparin and soy bean inhibitor, and the observation that fibrinogenolysis is slower in older rabbits than in the young, are all compatible with fibrinogenolysis being caused by activation of the plasmin system.

When blood is shed into a synovial joint the plasma fibrinogen becomes the substrate for two competing reactions, namely, conversion to fibrin by thrombin or fibrinogenolysis, probably by plasmin. The balance between these reactions is probably affected by the age and species of the subject, the severity of the joint injury and hence the concentration of thromboplastin liberated, and by the speed of entry of the blood, a slow ooze probably favouring fibrinogenolysis by allowing time for secondary anticoagulant factors to appear. The absence of thromboplastin in normal human joint tissues
(Astrup and Sjølin, 1958) also favours fibrinogenoly $\frac{\overline{0}}{-}$ sis rather than blood clotting.

The bulk of blood clot formed in a joint may? later be diminished by the lysis of red cells that seemsto be a constant feature of haemarthroses.

The cost of the experimental work on dogs was defrayecb by a grant from the Joint Standing Research Committee St. Mary's Hospital and Medical School.

The biochemical investigations were carried out with $\vec{P}$ the help, encouragement, and supervision of Dr. C. $\mathrm{H}=$ Lack in the Wellcome Research Laboratories, Institute్లు of Orthopaedics. The cost of this work was met in part by a grant from the Joint Clinical Research Committe of the Royal National Orthopaedic Hospital and the Institute of Orthopaedics.

This work forms part of a thesis accepted for the degrees of M.S. by the University of London.

\section{REFERENCES}

Astrup, T., and Müllertz, S. (1952). Arch. Biochem., 40, 346. _, and Sjølin, K. E. (1958). Proc. Soc. exp. Biol. (N.Y.) 97, 852

Biggs R., and Macfarlane, R. G. (1957). Human Blood Coagulation, and its Disorders, 2nd ed. Blackwell, Oxford.

Ha1 rold, A. J. (1960a). J. Bone Jt Surg., 42B, 226.

(1960b). Nature (Lond.), 186, 1057.

Jaffe, A. (1897). Arch. klin. Chir., 54, 69.

Julliard, C. (1902). Rev. Chir. (Paris), 25, 196.

Kling, D. H. (1938). The Synovial Membrane and the Synovial Fluido Medical Press, Los Angeles.

Lack, C. H. (1959). J. Bone Jt Surg., 41B, 384.

Niewiarowski, S., Latallo, Z., and Stachurska, J. (1959). Rev. Hémat $14,118$.

Ropes, M. W., and Bauer, W. (1953). Synovial Fluid Changes in Joint Disease. Harvard University Press, Camoridge, Massachusettso Stormorken, H. (1957). Brit. J. Haemat., 3, 299.

Triantaphyllopoulos, D. C. (1958). Canad. J. Biochem., 36, 249. 